http://jmscr.igmpublication.org/home/ ISSN (e)-2347-176x ISSN (p) 2455-0450 crossref DOI: https://dx.doi.org/10.18535/jmscr/v8i9.26

$\underline{\text { Research Article }}$

\title{
Evaluation of Non-Invasive Bio-Markers, AST to Platelet Ratio Index (APRI) in the Assessment of Liver Fibrosis
}

\author{
Authors \\ Himanshu Goyal ${ }^{1}$, Subodh Prakash Katariya ${ }^{2 *}$, Ila Pahwa ${ }^{3}$ \\ ${ }^{1}$ PG-JR-3, Department of Medicine, Muzaffarnagar Medical College, Muzaffarnagar (U.P.) \\ ${ }^{2}$ Professor, Department of Medicine, Muzaffarnagar Medical College, Muzaffarnagar (U.P.) \\ ${ }^{3}$ Professor and HOD, Department of Medicine, Muzaffarnagar Medical College Muzaffarnagar (U.P.) \\ *Corresponding Author \\ Dr Subodh Katariya
}

\begin{abstract}
Introduction: For diagnosis of staging of liver fibrosis, the liver biopsy is the only recognized gold standard technique. The aspartate transaminase to platelet ratio index (APRI) has been proposed as a noninvasive and readily available tool for the assessment of liver fibrosis.

Method: The present prospective study was carried out in the Department of Medicine, Muzaffarnagar Medical College, Muzaffarnagar.

Result: In this study, we have included 203 patients, out of which $72 \%$ were male and $28 \%$ were female. Viral etiology noted in 48.3\%, alcoholism in 5.9\% and other etiology noted in 45.8\%. With APRI cut-off 1 the specificity, sensitivity and positive predictive value to detect advanced fibrosis was $87 \%, 42 \%, 75 \%$ respectively.

Conclusion: The present showed that the APRI is a specific non-invasive biomarker to detect advanced fibrosis but liver elastography remains the best available non-invasive tool to diagnosis liver low grade and advanced fibrosis due to the poor sensitivity of these markers.

Keywords: APRI, liver fibrosis, Aspartate Transaminase, Platelet count.
\end{abstract}

\section{Introduction}

The incidence of chronic liver disease is rising so the need to stage liver disease and fibrosis accurately is paramount as it helps guide therapy and informs prognosis. ${ }^{[1]}$ Liver biopsy is a flawed gold standard, associated with morbidity and mortality. ${ }^{[2]}$ Several non-invasive biochemical tests for eg. Fibro Test ${ }^{[3]}$, hepascore, transient electrography ${ }_{2}^{[4]}$ fibrospect, forns index, AAR, ELF etc. are currently in use $\mathrm{e}^{[5-6]}$, requiring complex calculations and expensive biochemical assays.
The assessment serum markers are in constant evolution presenting an alternative to liver biopsy. These markers are classified as direct, which represent extracellular matrix components (reflecting the pathophysiology of liver fibrogenesis); and indirect (or class II) which use routine laboratory data (reflecting the consequences of the liver damage). Direct and indirect markers may be use alone as well as in combination to produce composite scores. ${ }^{[7]}$ Application of simple non-invasive test to assess fibrosis could provide a safe way of identifying 
patients in greatest need of intervention and of monitoring response to therapy. Hence the aim of our study was $\mathrm{t}$ o evaluate fibrosis using noninvasive bio-markers APRI to validate them as primary tools against liver elastography for diagnosis liver fibrosis. In my study I have shown that Uses of APRI with a cut off $>1$.

\section{Methods}

The Present study was carried out in the Department of Medicine, Muzaffarnagar Medical College, Muzaffarnagar from June 2017 to June 2018. 203 patients of age group 16-82 were selected from our clinic with chronic liver disease (compensated cirrhosis and chronic hepatitis) of any etiology-alcohol, viral, autoimmune and cryptogenic were included in the study with informed consent.

\section{Exclusion Criteria}

Pregnant women, Patient with acute hepatitis, acute or chronic liver failure and decompensated chronic liver disease were excluded from the study.

Method: Appropriate baseline tab and radiological parameters were done. AST, ALT was calculated using International Federation of Clinical Chemistry without pyridoxal phosphate (P-5-P) Kinetics. Platelets count was calculated by Culter electronic counter and by manual count. APRI was calculated using the formula -
(AST/upper limit of normal)/Platelet count (109/L) x 100. Liver elastography was done using Toshiba APLIO 500 ultrasound and ARFI was also generated.

\section{Result}

In the present study, we have enrolled 203 patients of age group 16-82 years. Out of 203 patients, $72 \%$ were male and $28 \%$ were female.

Viral etiology noted in $48.3 \%$, alcoholism in $5.9 \%$ and other etiology noted in $45.8 \%$. With APRI cut-off 1 the specificity, sensitivity and positive predictive value to detect advanced fibrosis was $87 \%, 42 \%, 75 \%$ respectively. The values of noninvasive indices were compared with the results of liver elastography in order to detect advanced fibrosis (F3 and F4).

Table: No- 1 Showing Baseline Characteristics of All Participants

\begin{tabular}{|l|c|c|c|c|c|}
\hline S.No & Variables & Minimum & Maximum & Mean & $\begin{array}{c}\text { Standard } \\
\text { Deviation }\end{array}$ \\
\hline 1 & $\begin{array}{c}\text { Age } \\
\text { (Years) }\end{array}$ & 16 & 81 & 46.72 & 13.57 \\
\hline 2 & $\begin{array}{c}\text { SGOT } \\
\text { (IU/L) }\end{array}$ & 11 & 257 & 44.61 & 32.52 \\
\hline 3 & $\begin{array}{c}\text { SGPT } \\
\text { (IU/L) }\end{array}$ & 9 & 218 & 47.74 & 39.03 \\
\hline 4 & Platelets & 37 & 532 & 178.95 & 60.54 \\
\hline 5 & Albumin & 1.6 & 5.3 & 3.91 & 0.56 \\
\hline 6 & Bilirubin & 2 & 3.2 & 0.98 & 0.41 \\
\hline 7 & TLC & 2400 & 15400 & 6593 & 2502.6 \\
\hline 8 & APRI & 0 & 10.20 & 0.95 & 1.09 \\
\hline
\end{tabular}
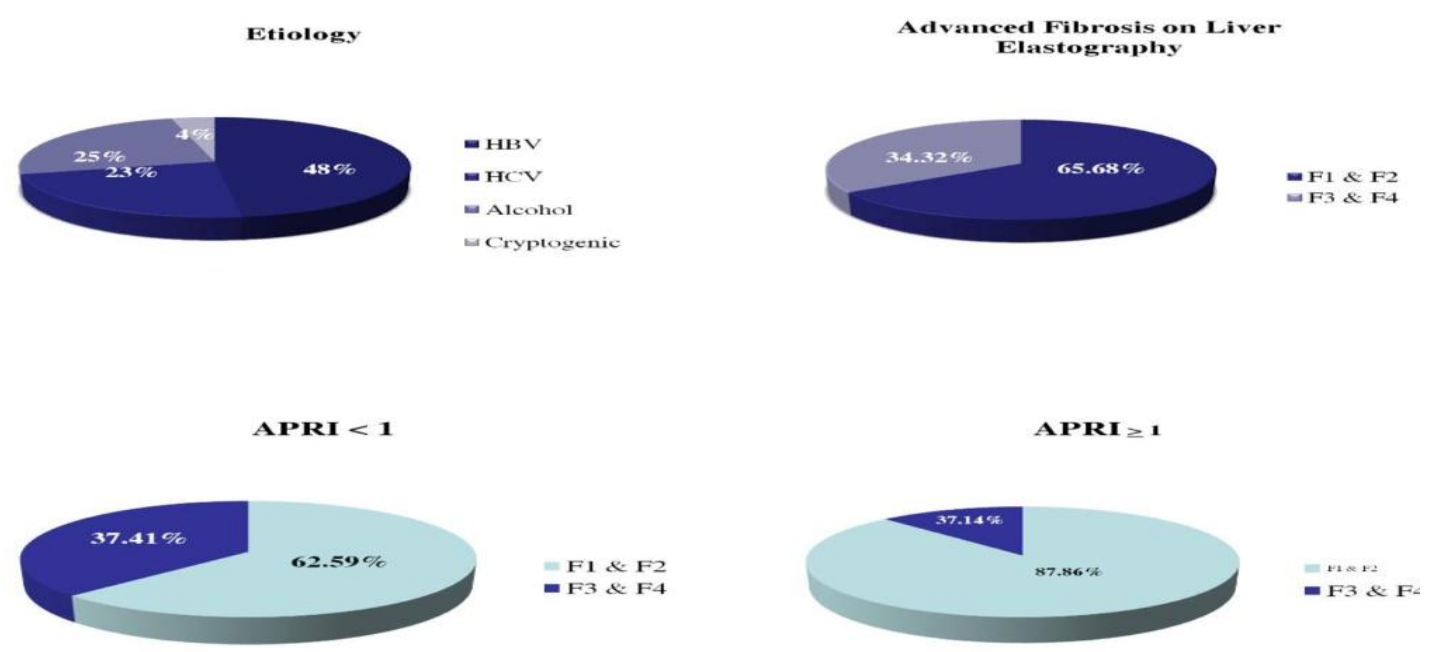


\section{Discussion}

Non-invasive assessment of liver fibrosis is a focus of continuing interest to find a marker that is cost-effective, easy to perform with standard laboratory tests, accurately reflective of the stage of hepatic fibrosis, and valid over a wide patient population. ${ }^{[8]}$ The APRI is a simple test that can reliably differentiate mild from significant fibrosis in chronic HCV patients. ${ }^{[9]}$ The liver cirrhosis due to chronic HCV infection and non-alcoholic fatty liver disease are the leading cause of deaths. Fibrosis in connective tissue followed by its extension in hepatic tissue in HCV infection is an evidence of cirrhosis. ${ }^{[10-11]}$

In the present study we evaluated the accuracy of APRI as non-invasive marker for liver cirrhosis. The result of our study showed that APRI is cheap and accurate non-invasivae marker. As the cost of both the test is very low and due to the easily availability of these two variables, makes APRI a useful and simple bedside test.

To evaluate the fibrosis stage, Liver biopsy has been advised as a gold standard, an invasive, costly and serious consequences like soreness, bile leakage, infection, severe right hypochondriac pain, haemorrhages and also liver biopsy requires expert hands for sampling and is not cost-effective for the patients. ${ }^{[12]}$

Petersen et al in their study concluded that the APRI and $\mathrm{ELF}^{\mathrm{TM}}$ tests are essentially equivalent in the prediction of mild and significant fibrosis in chronic hepatitis $\mathrm{C}$ and complimentary in clinical utility. ${ }^{[13]}$

Jain et al in their study observed that, APRI A simple parameter which consisting of easily availability of AST and Platelet count and concluded that APRI can be a better predictor for liver cirrhosis with high degree of accuracy. ${ }^{[14]}$

However, further prospective studies are needed to validate the APRI in a larger number of patients in other institutes.

\section{Conclusion}

APRI had specificity, sensitivity and PPV of $82.87 \%, 42 \%, 75 \%$ respectively to diagnose advanced fibrosis in our study. APRI is specific non-invasive biomarkers to detect advanced fibrosis but liver elastography remains the best available non-invasive tool to diagnosis liver low grade and advanced fibrosis due to the poor sensitivity of these markers.

\section{References}

1. Schuppan D, Afdhal NH. Liver Cirrhosis. Lancet 2008;371(9615):838:51.

2. Sharma S, Kahlili K, Nguyen GC. NoInvasive Diagnosis of Advanced Fibrosis and Cirrhosis.World J Gastroenterol 2014 7;20(45):16820-30.

3. Poynard $\mathrm{T}$, Imbert-Bismut $\mathrm{F}$, Munteanu M, Messous D, Myers RP, Thabut D, et al. Overview of the diagnostic value of biochemical markers of liver fibrosis (Fibro Test, HCV Fibro Sure) and necrosis (Acti Test) in patients with chronic hepatitis C. Comp Hepatol. 2004;23:3-8.

4. Forestier J, Dumortier J, Guillaud O, Ecochard M, Roman S, Boillot O, et al. Noninvasive diagnosis and prognosis of liver cirrhosis: a comparison of biological scores, elastometry, and metabolic liver function tests. Eur J Gastroenterol Hepatol. 2010;22:532-40.

5. Lesmana CRA, Salim S, Hasan I, Sulaiman AS, Gani RA, Pakasi LS, et al. Diagnostic accuracy of transient elastography (FibroScan) versus the aspartate transaminase to platelet ratio index in assessing liver fibrosis in chronic hepatitis B: the role in primary care setting. J Clin Pathol. 2011;64:916-20.

6. Fallatah HI. Noninvasive biomarkers of liver fibrosis: An Overview. Advances in Hepatology. 2014;2014:1-15.

7. Papastergiou V, Tsochatzis E, Burroughs AK. No-Invasive Assessment of Liver Fibrosis. Ann Gastroenterol 2012;25(3): 218:31.

8. Fabris C, Smirne C, Toniutto P, Colletta C, Rapetti R, Minisiniet R et al. Usefulness of 
six non-proprietary indirect markers of liver fibrosis in patients with chronic hepatitis C. Clin Chem Lab Med. 2008;46:253-59.

9. Paggi S, Colli A, Fraquelli M, Vigano M, Poggio PD, Facciotto C, et al. A noninvasive algorithm accurately predicts advanced fibrosis in hepatitis $\mathrm{C}$ : a comparison using histology with internalexternal validation. J Hepatol. 2008;49: 564-71.

10. Attaullah S, Khan S, Ali I. Hepatitis C virus genotypes in Pakistan: a systemic review. Virol J 2011;8.doi:10.1186/1743422X-8-433.

11. Ahmad W, Ijaz B, Javed FT, Gull S, Kausar H, Sarwar MT, et al. A comparison of four fibrosis indexes in chronic $\mathrm{HCV}$ : development of new fibrosis-cirrhosis index (FCI). BMC Gastroenterol 2011; 11:44.doi:10.1186/1471-230X-11-44.

12. Schmid P, Bregenzer A, Huber M, Rauch A, Jochum W, Mullhaupt B, et al. Progression of liver fibrosis in $\mathrm{HIV} / \mathrm{HCV}$ co-infection: assessment methods and liver biopsy. PLoS One 2015;10:e0138838.

13. Petersen JR, Stevenson HL, Krishna SK, Naniwadekar A, Parkes J, Cross R, et al. Evaluation of the APRI (AST, platelet ratio index) and ELFTM (Enhanced Liver Fibrosis) tests to detect significant fibrosis due to chronic hepatitis $\mathrm{C}$.

14. Jain P, Tripathi BK, Gupta B, Bhandari B, Jalan D. Evaluation of Aspartate Aminotransferase-to-Platelet Ratio Index as a Non-Invasive Marker for Liver Cirrhosis. J Clin Diagn Res 2015; Nov 9(11):22-4. 